\section{SAT0601 RELATIONSHIP BETWEEN BODY MASS INDEX AND NAILFOLD CAPILLAROSCOPY IN PRIMARY AND SECONDARY RAYNAUD'S PHENOMENON}

R. Tirri ${ }^{1}$, M. Barba ${ }^{2}$, R. Formica ${ }^{2}$, R. Irace3, F. Ciccia ${ }^{2} .{ }^{1}$ Rheum, Precision Medicine, Naples, Italy; ${ }^{2}$ Rheumatology Unit, Precision Medicine, Naples, Italy; ${ }^{2}$ Rheumatology Unit, Precision Medicine, Naples, Italy

Background: Raynaud's phenomenon (RP) is a diffuse clinical manifestation (3-5\% of general population) RP is often secondary to autoimmune systemic diseases, while the condition is classified primary if no underlying disorders can be found. A lower body mass index (BMI) was associated with a greater risk of developing RP, perhaps due to greatest sensivity to cold temperatures

Objectives: The objective of our study was to evaluate the association of BMI with clinical and capillaroscopic features in primary and secondary RP.

Methods: Consecutive patients at the first access to a Rheumatology Outpatient Clinic over a 13 months period were screened to RP; nailfold videocapillaroscopy (NVC)was carried out and qualitative and quantitative assessment was performed. Diagnosis of RP was defined in patients who identified color pictures of witnessed attacks.Patients enrolled were screened for secondary causes of RP. RP was classified as primary when no abnormalities were found. Weight and height were collected in clinical records and patients were divided in 3 groups according to their BMI: underweight $(B M l<18.5 \mathrm{~kg} / \mathrm{m} 2)$, normal weight (BMI 18.5-25 kg/m2), and overweight (BMI >25 kg/m2). Chi-square test to compare categorical variable and Parametric Student t-test to comparing mean values of normally distributed data were used. $p<0.05$ was considered to be statistically significant.

Results: RP was diagnosticated in 100 of 1416 patients (7.06\%). Of these, 73 $(10 \mathrm{M}, 63 \mathrm{~F})$ accepted to undergo NVC. An autoimmune disease was found in 35 patients $(47.9 \%)$, of which 2 were underweight, 14 normal weight and 19 overweight. Of 38 patients with primary RP, 3 were underweight, 23 normal weight and 12 overweight. BMI was significantly higher in secondary RP $(p=0.03)$. Overweight patients with secondary RP were older $(p=0.01)$, but with a disease duration not statistically significant longer $(p=0.26)$. In secondary RP, avascular areas and neoangiogenesis were founded only in overweight patients. Moreover,in secondary RP overweight was correlated with decreased capillary density $(p=0$ 04). There was not association between BMI and capillaroscopic abnormalities in primary RP.

Conclusion: In our study BMI was correlated with microvascular changes only in patients with secondary RP. Our findings may suggests a role for obesity in the microcirculatory disfunction in the autoimmune diseases. Further studies are needed to generalize results and to find a causative role.

References:

[1] Lemmens HA. Vasa Suppl. 1987;18:10-4.

[2] Le RoyEC,MedsgerTA.ClinExpRheumatol 1992;10;485-8

[3] O Keeffe ST et al Journal Rheumat 1992:19:1415-7

[4] Fraenkel L. Curr Rheumatol Rep. 2002;4(2): 123-8.

[5] MaundrellA et al NY:Spinger New York 2015 p 21-35

[6] Giurgea G. et al Arthritis Rheumatol Vol. 67, No. 1, January 2015, pp 238-242

[7] A.Eman et al. Ab / Atherosclerosis 287 (2019) e123-288

Disclosure of Interests: Rosella Tirri: None declared, Marco Barba: None declared, Ranieri Formica: None declared, Rosaria Irace: None declared, francesco ciccia Grant/research support from: pfizer, novartis, roche, Consultant of: pfizer, novartis, lilly, abbvie, Speakers bureau: pfizer, novartis, lilly, abbvie DOI: 10.1136/annrheumdis-2020-eular.4367

\section{SAT0602 \\ INCIDENCE OF INFLAMMATORY ARTHRITIS BEFORE AND AFTER THE INTRODUCTION OF NEW CLASSIFICATION CRITERIA: A SYSTEMATIC REVIEW}

E. Van Delft ${ }^{1}$, M. J. Abdelkadir ${ }^{1}$, H. Den Braanker ${ }^{1,2}$, J. Hazes ${ }^{2}$, D. Lopes Barreto ${ }^{1}$, A. Weel $l^{1,2,3} .{ }^{1}$ Maasstad Hospital, Rheumatology, Rotterdam, Netherlands; ${ }^{2}$ Erasmus Medical Center, Rheumatology, Rotterdam, Netherlands; ${ }^{3}$ Erasmus School of Health Policy \& Management, Health Technology Assessment, Rotterdam, Netherlands

Background: Hallmark in treatment of inflammatory arthritis (IA); rheumatoid arthritis (RA), axial spondyloarthritis (axSpA) and psoriatic arthritis (PsA), is to treat early with intensive regimens to induce remission and prevent disability on the longer term $[1,2,3]$. To be able to start treatment in an earlier stage of the disease, around a decade ago new classification criteria sets were introduced; 2010 ACR/EULAR for RA, 2009 ASAS for axSpA and 2006 CASPAR criteria for PsA. Compared to old criteria sets for RA, axSpA and PsA these new ones cover a broader spectrum of early disease features [2]. Although classification criteria are not developed for diagnosing, they are widely adopted for use as aids in diagnosing IA [4]. Therefore, the new criteria sets might have led to an increased reported incidence of IA [5].
Objectives: To determine whether the reported incidence of IA has changed after introduction of the new classification criteria this systematic review has been conducted.

Methods: A systematic literature search was conducted using Embase, Medline Ovid, Cochrane Central and Web of Science from database inception to September 2019. For this study only articles that addressed the incidence of IA in adult referrals towards the rheumatologist were included (Fig. 1). A meta-analysis was performed to compare the pooled estimates and $95 \%$ confidence intervals (Cl) for the incidence of IA before and after the introduction of new classification criteria.
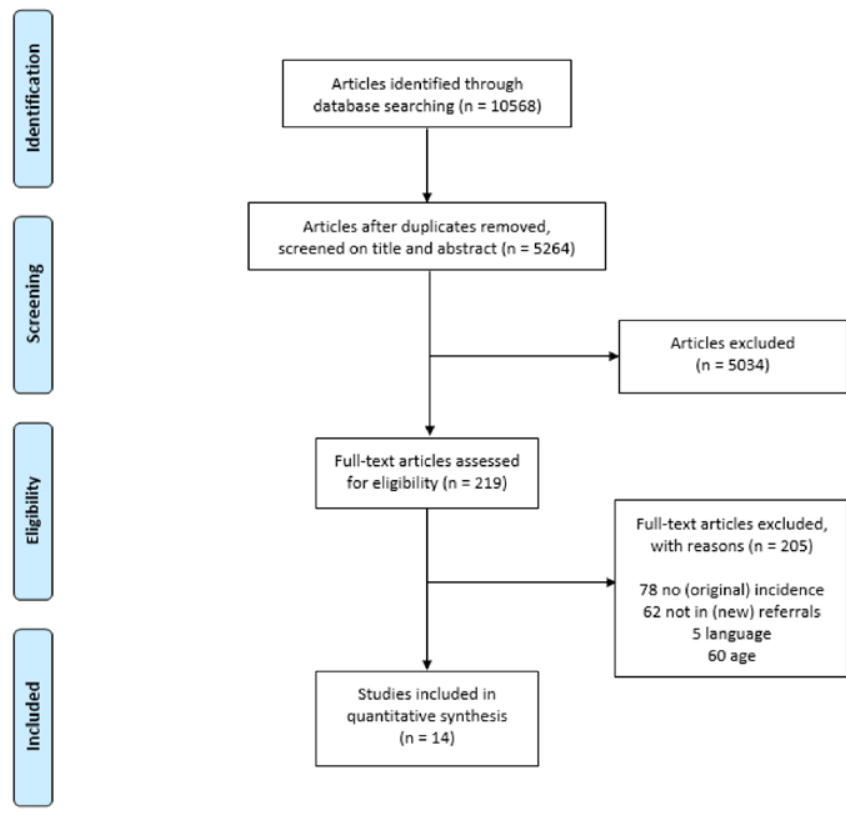

Figure 1. PRISMA flow diagram of study selection

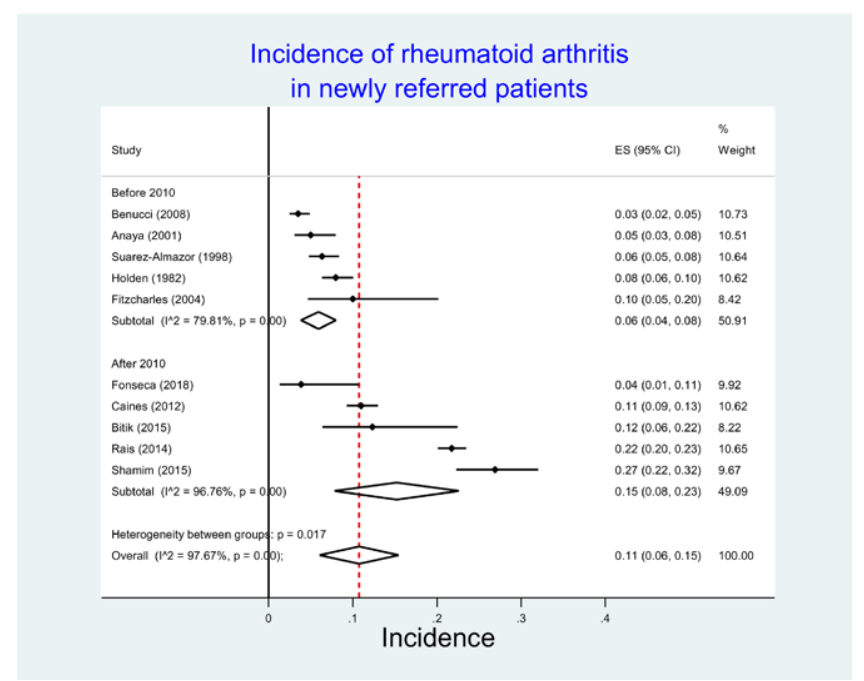

Graph 1. Forest plot of incidence of rheumatoid arthritis compared before and after introduction of new classification criteria in 2010

Results: A total of 14 studies reporting on the incidence of RA $(n=10)$, axSpA $(n=7)$ and PsA $(n=4)$ in adults newly referred towards the rheumatologist were included. Pooled incidences were for RA $11 \%$ (95\% Cl 6-15\%); for axSpA $4 \%$ (95\% Cl 3-5\%); and for PsA 4\% (95\% Cl 3-5\%).

Graph 1 and 2 show a significant increase in incidence of $9 \%$ for RA ( $p=0.019)$ and $2 \%$ for axSpA $(p=0.006)$ after the introduction of the new classification criteria for respectively RA and axSpA. For PsA only one study was included reporting on the incidence of PsA in rheumatology centres before 2006, hence pooled estimates before and after introduction of new criteria could not be compared. 
Overall there was a high intragroup heterogeneity $(p=0.00)$ caused by geographical area and study design.

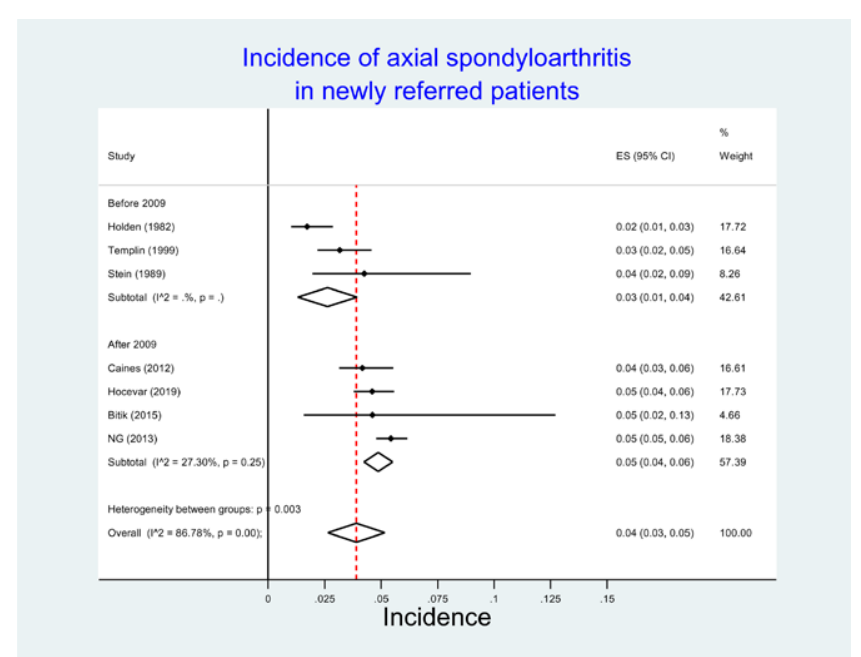

Graph 2. Forest plot of incidence of axial spondyloarthritis compared before and after introduction of new classification criteria in 2009

Conclusion: Rheumatoid arthritis is the most frequent IA diagnosis in newly referred patients, of which the incidence increased over time up to $15 \%$. The introduction of new classification criteria might have introduced higher incidence rates for IA, although heterogeneity could have influenced the results.

References:

[1] Monti S, Montecucco C, Bugatti S, Caporali R. Rheumatoid arthritis treatment: the earlier the better to prevent joint damage. RMD Open, 2015. 1

[2] Dubash S, McGonagle D, marzo-Ortega $\mathrm{H}$. New advances in the understanding and treatment of axial spondyloarthritis: from chance to choice. Ther Adv Chronic Dis, 2018. 9(3):77-87

[3] McLaughlin M, Ostör A. Early treatment of psoriatic arthritis improves prognosis. Practitioner, 2014. 258:21-4

[4] Rudwaleit M, Taylor WJ. Classification criteria for psoriatic arthritis and ankylosing spondylitis/axial spondyloarthritis. Best Pract Res Clin Rheumatol, 2010. 24(5):589-604

[5] Van der Linden MPM, Knevel R, Huizinga TWJ, van der Helm-van Mil AHM. Classification of rheumatoid arthritis: comparison of the 1987 American College of Rheumatology criteria and the 2010 American College of Rheumatology/European League Against Rheumatism criteria. Arthritis Rheum, 2011. 63(1):37-42

Disclosure of Interests: None declared

DOI: 10.1136/annrheumdis-2020-eular.2136

\section{SAT0603 SYSTEMIC SCLEROSIS IS AN INDEPENDENT RISK FACTOR FOR ISCHEMIC HEART DISEASE WITH AN ADDITIONAL RISK IN THOSE POSITIVE FOR CERTAIN ANTI-PHOSPHOLIPID ANTIBODIES: A VERY LARGE CASE-CONTROL STUDY}

A. Watad ${ }^{1}$, D. Mcgonagle ${ }^{2}$, N. L. Bragazzi ${ }^{3}$, D. Comanesther ${ }^{4}$, A. Cohen $^{4}$, M. Lidar ${ }^{5}$, H. Amital' . 'Sheba Medical Center, Department of Medicine B', Rheumatology Unit, Ramat Gan, Israel; ${ }^{2}$ Leeds Musculoskeletal Biomedical Research Unit, Leeds, United Kingdom; ${ }^{3}$ University of Genoa, Genova, Italy; ${ }^{4}$ Clalit Health Services, Tel Aviv-Yafo, Israel; ${ }^{5}$ Sheba Medical Center, Rheumatology Unit, Ramat Gan, Israel

Background: A higher prevalence of ischemic heart disease (IHD) in patients with systemic sclerosis (SSc) was reported. However, contrasting findings were published concerning the role of SSc-related autoantibodies in IHD risk which remains controversial.

Objectives: The current study explored the link between SSc and IHD, impact of putative links on SSc mortality and the role of SSc-related and antiphospholipid autoantibodies in disease associated IHD.

Methods: A large cohort study utilising the Clalit-Health-Service (CHS) database was conducted on 2,431 SSc patients and 12,710 age- and sex matched controls. The proportion of IHD was compared between patients diagnosed with SSc and age- and gender-matched controls. The role of
SSc-linked and antiphospholipid autoantibodies in disease associated IHD was assessed.

Results: The rate of IHD was significantly higher in SSc than controls $(20.4 \%$ vs $15.0 \%, \mathrm{p}<0.001)$. At the multivariate analysis, SSc was an independent predictor of IHD with an OR of $1.91(95 \% \mathrm{Cl} 1.57-2.31, \mathrm{p}<0.0001)$. SSc patients with IHD had a higher mortality rate with an $\mathrm{HR}$ of $2.67(95 \% \mathrm{Cl} 2.03-3.53, \mathrm{p}<0.0001)$ than those without IHD. SSc patients with positive anti-beta2GPI (IgM-isotype) or anti-cardiolipin (aCL) (IgA-isotype) exhibited a higher risk of IHD than SSc patients without these antibodies with an OR 1.89 (95\% 1.04-3.45, $\mathrm{p}=0.0369)$ and OR of 3.72 (95\% 1.25-11.11, $p=0.0184)$, respectively.

Conclusion: Patients with SSc are at higher risk for developing IHD with an additional risk for the latter in those positive for aCL or anti-beta2GPI. A high degree of suspicion is needed during routine patient follow-up and pre-emptive screening should be considered.

Disclosure of Interests: Abdulla Watad: None declared, Dennis McGonagle Grant/research support from: Janssen Research \& Development, LLC, Nicola Luigi Bragazzi: None declared, Doron Comanesther: None declared, Arnon Cohen: None declared, Merav Lidar: None declared, Howard Amital: None declared

DOI: 10.1136/annrheumdis-2020-eular.6253

\section{SAT0604 \\ FAST FOOD HABITS AND SERUM URATE CHANGE IN YOUNG ADULTS: 15-YEAR PROSPECTIVE} ANALYSIS

$\underline{\text { C. Yokose }}^{1,2}$, L. Lu ${ }^{3}$, N. Mccormick ${ }^{1,2,3}$, J. Choi ${ }^{1}$, Y. Zhang ${ }^{1,2}$, H. Choi ${ }^{1,2}$ ${ }^{1}$ Massachusetts General Hospital, Division of Rheumatology, Allergy, and Immunology, Boston, United States of America; ${ }^{2}$ Massachusetts General Hospital, Clinical Epidemiology Program, Mongan Institute, Boston, United States of America; ${ }^{3}$ Arthritis Research Canada, Richmond, Canada

Background: Fast food consumption has strong positive associations with weight gain and insulin resistance. ${ }^{1}$ Obesity and insulin resistance are in turn strongly associated with elevated serum urate (SU) levels, largely mediated by insulin's anti-uricosuric ability. ${ }^{2}$

Objectives: To investigate the relation between fast food consumption and changes in SU over a 15-year period among young black and white adults in the United States.

Methods: Participants for the CARDIA study included 3,122 young (age 18-30 years in 1985-86) black and white adults in the United States who were followed up with repeated dietary and clinical assessments and had both baseline and year $15 \mathrm{SU}$ measurement available. Frequency of fast food consumption (fast food frequency, FFF) was quantified on a semicontinuous scale and classified as $<1,1-2$, or $>2$ times per week. We used multivariable linear regression models to investigate the association of FFF at baseline as well as change in FFF with 15-year changes in SU.

Results: Our analysis included data from 3,122 subjects who had SU data available both at baseline and year 15 (Table 1). After adjustment for age, sex education, baseline height and weight, and baseline SU, baseline FFF (defined as 3 times per week year 0 differences between participants) was independently associated with increases in SU among both black (beta=0.11, $p=0.04$ ) and white (beta $=0.11, p=0.01$ ) individuals (Table 2). Change in FFF (defined as 3 times a week 15-year change within participants) was also independently associated with increases in SU among white (beta $=0.09, p=0.01$ ) individuals but not blacks (beta $=0.03, p=0.93$ ) (Table 2). There was a significant correlation between weight change and SU change (correlation coefficient $0.34, p<0.001$ ) Figure 1 depicts the joint associations of year 0 FFF and 15-year changes in FFF with change in weight. Compared to the average 15-year SU change among participants with baseline FFF $<1$ time per week and 15-year FFF change $<0$ time per week, those with high FFF at both baseline and follow-up had an extra $0.21 \mathrm{mg} / \mathrm{dL}$ increase (i.e., $75 \%$ of overall population SU increase over 15 years [0.28 mg/dL]) in SU during that time. After adjusting for covariates in model 2 , change in weight (beta $=0.03, p<0.001$ ) and homeostasis model fo insulin resistance $(\mathrm{HOMA})$ (beta $=0.05, \mathrm{p}<0.001$ ) remained significantly associated with SU change.

Table 1. Participant Characteristics

\begin{tabular}{lcc}
\hline Characteristic & Blacks $(\mathrm{n}=1468)$ & Whites $(\mathrm{n}=1654)$ \\
\hline Age, years (year 0) & $24.4(3.8)$ & $25.6(3.3)$ \\
Male (\%) & 44 & 48 \\
Weight, kg (year 0) & $72.8(16.7)$ & $70.0(14.0)$ \\
Weight, kg (year 15) & $87.9(20.9)$ & $80.7(18.6)$ \\
Serum urate, $\mathrm{mg} / \mathrm{dL}$ (year 0) & $5.1(1.4)$ & $5.4(1.4)$ \\
Serum urate, $\mathrm{mg} / \mathrm{dL}$ (year 15) & $5.6(1.4)$ & $5.5(1.4)$ \\
\hline
\end{tabular}

All values reported as mean $(S D)$ unless otherwise noted. 